\title{
APLICAÇÃO DE MODELO MATEMÁTICO ÀS PROPRIEDADES REOLÓGICAS DE GÉIS MISTOS DE AGAROSE
} E DE GOMA GUAR

Cristina T. Andrade, Rosangela B. Garcia ${ }^{a}$

Instituto de Macromoléculas Professora Eloisa Mano - Universidade Federal do Rio de Janeiro - CP 68525 - 21945 -970 Rio de Janeiro - RJ

Marcos W. N. Lobão

Programa de Engenharia Química - COPPE - Universidade Federal do Rio de Janeiro - CP 68502 - 21945-970 - Rio de Janeiro - RJ

Recebido em 15/12/95; aceito em 24/10/96

\begin{abstract}
APPLICATION OF A MATHEMATICAL MODEL TO THE RHEOLOGICAL PROPERTIES OF AGAROSE-GUAR GUM MIXED GELS. Certain biopolymers are capable of forming physically crosslinked gels in aqueous medium, stabilized by forces such as Coulombic, charge transfer, hydrogen bonding, dipole-dipole, van der Waals, and hydrophobic interactions. The mathematical description of these physical networks are difficult, but should contribute to a better understanding of the gelling process. The Clark and Ross-Murphy model was applied to experimental data for agarose-guar gum mixed systems, in which only agarose is the gelling polysaccharide. A computational routine based on the statistical maximum likehood principle was employed to estimate the $f, \mathrm{~K}$ and a characteristic parameters. Statistical t-test and F-test were used to analyse the set of parameters.
\end{abstract}

Keywords: agarose-guar gum mixed gels; Clark and Ross Murphy model.

\section{INTRODUÇÃO}

$\mathrm{Na}$ área de polímeros, a pesquisa de sistemas gelificados desperta interesse, devido às suas aplicações em diversos segmentos industriais. Algumas definições para o processo de gelificação foram propostas ${ }^{1,2}$. Qualitativamente, os sistemas gelificados apresentam como característica comum uma estrutura macromolecular tridimensional contínua, preenchida ou não com excesso de solvente, mas capaz de imprimir propriedades elásticas ao sistema ${ }^{1}$.

Os géis poliméricos podem ser subdivididos em três classes principais. A primeira engloba os géis químicos, assim denominados devido à natureza covalente das ligações cruzadas, que dão origem à rede tridimensional. Essas redes são sintetizadas através de diversas rotas e constituem-se em macromoléculas de peso molecular infinito, caracterizadas por um tempo de relaxamento infinito e por um módulo de equilíbrio ${ }^{3}$. Uma segunda classe de gel, na verdade pseudo-gel, é formada a partir de interações topológicas ou entrelaçamentos de cadeias poliméricas fundidas ou em solução concentrada ${ }^{4}$. A adição de excesso de solvente permite a diferenciação visual entre os tipos citados de rede. Enquanto os géis químicos apenas sofrem inchamento, os pseudo-géis transformam-se em soluções mais diluídas.

Alguns biopolímeros e polímeros sintéticos são capazes de fornecer géis físicos, com características especiais, que os situam numa classe intermediária. Nesses géis, a rede tridimensional é formada por ligações cruzadas não-covalentes, estabilizadas por forças tão variadas como as eletrostáticas, pontes de hidrogênio, ligações hidrofóbicas, interações dipolodipolo, etc. Além disso, o número e a posição dessas ligações cruzadas flutuam com o tempo e a temperatura ${ }^{5}$.

a. Endereço atual: Departamento de Química - Universidade Federal do Rio Grande do Norte - Campus da Universidade - 59072-970 Natal - RN

b. Endereço atual: Departamento de Engenharia Química - Universidade Federal de Sergipe - CP 353 - 49100-000 - Aracaju - SE
No caso de certos biopolímeros, como a agarose e a $\kappa$ carragenana, géis físicos são obtidos a partir de soluções aquosas de concentrações relativamente baixas. A diminuição da temperatura ou a adição de contra-íons induzem à formação da rede e à imobilização de volume considerável de água. Inicialmente, a macromolécula passa da conformação desordenada para uma conformação mais ordenada. Devido a fatores estruturais $^{6}$, regiões da cadeia permanecem em conformação desordenada. $\mathrm{O}$ processo de gelificação progride com a associação posterior de regiões ordenadas, que passam então a formar as chamadas zonas de junção.

A agarose é um polissacarídeo gelificante, extraída no Brasil de Gracilaria verrucosa, uma espécie de alga vermelha (Rhodophyceae), e utilizada pelas indústrias alimentícia e farmacêutica. Sua estrutura química consiste de unidades (1Ø3)- $\beta$ D-galactopiranosila e (1Ø4)-3,6-anidro- $\alpha$-L-galactopiranosila, ligadas alternadamente. A goma guar é um polissacarídeo neutro, no qual à cadeia de (1Ø4)- $\beta$-D-manopiranosila, resíduos de $\alpha$-D-galactopiranosila encontram-se ligados, através das posições 1- e 6-, respectivamente. Originária do Paquistão, esta goma é extraída do endosperma de sementes de Cyamopsis tetragonolobus. Como outras galactomananas, a goma guar não forma géis físicos verdadeiros e é empregada como espessante.

A gelificação da agarose em sistemas aquosos tem sido descrita por mecanismos diversos. No primeiro deles, a transição sol-gel seria resultante da associação de hélices duplas ${ }^{7}$. Alguns autores propuseram que o processo deveria passar-se através de uma decomposição espinodal ${ }^{8}$. Foi verificado que a formação de rede tridimensional em soluções muito diluídas de agarose ocorre pela conexão de domínios ricos em polímero9-11, provavelmente devido a um processo espinodal de separação de fases ${ }^{9,10}$. Em soluções mais concentradas, a formação de gel é favorecida antes mesmo da separação de fases ${ }^{9-11}$.

Soluções e géis de hidrocolóides e os produtos alimentícios aos quais eles são incorporados apresentam caráter sólido e caráter líquido; isto é, são viscoeláticos ${ }^{12}$. Na verdade, praticamente todos os materiais comportam-se tanto como sólidos e como líquidos, dependendo da escala de tempo do experimento aos quais são submetidos ${ }^{13}$. 
Géis físicos de hidrocolóides, particularmente de agarose e de misturas de agarose e goma guar podem ser moldados, a partir do resfriamento de soluções relativamente concentradas, sob a forma de cilindros que sustentam o próprio peso e mantêm a forma durante semanas ${ }^{12}$. Os géis físicos são capazes de armazenar o trabalho empregado em sua deformação e de recuperar a sua forma original, isto é, apresentam elasticidade, propriedade essencial de um sólido ${ }^{14}$. Apesar do emprego crescente de experimentos oscilatórios na caracterização de materiais, experimentos não-oscilatórios ainda são os métodos mais utilizados na caracterização de materiais tanto sólidos como líquidos.

As propriedades mecânicas têm sido utilizadas freqüentemente na elucidação da estrutura molecular de géis de biopolímeros, pois fornecem dados representativos sobre a conectividade da rede polimérica tridimensional formada no gel ${ }^{15}$.

A partir da base teórica estabelecida por Flory $^{16}$ e Stockmayer ${ }^{17}$, modelos matemáticos foram sugeridos para a descrição de géis químicos ${ }^{18}$, nos quais a rede é formada por ligações covalentes. Nesses casos, várias técnicas podem ser utilizadas para a determinação do grau de ligações cruzadas ${ }^{19-21}$. Para géis físicos, nos quais o número e a posição das ligações cruzadas físicas ou zonas de junção flutuam em função do tempo e da temperatura ${ }^{3,22-24}$, a descrição matemática torna-se mais complexa. Apesar da definição ambígüa, modelos teóricos foram propostos por Hermans ${ }^{25}$ e por Clark e Ross-Murphy ${ }^{26}$, com o intuito de explicar a formação de géis físicos.

No modelo de Clark e Ross-Murphy ${ }^{26}$, o módulo de cisalhamento no equilíbrio, $\mathrm{G}$, é relacionado à concentração de polímero, C, pela equação 1 .

$$
\mathrm{G}=\frac{\operatorname{aRT}(f-1) \alpha(1-v)^{2}(1-\beta)}{2 \mathbf{K}(\boldsymbol{f}-2)^{2}} \frac{\mathrm{C}}{\mathrm{C}_{\mathrm{o}}}
$$

onde $\mathrm{R}$ é a constante dos gases, $\mathrm{T}$ é a temperatura absoluta, $\mathrm{C}_{\mathrm{o}}$ é a concentração crítica de gelificação, $\boldsymbol{f}$ é a funcionalidade (número de sítios ou grupos funcionais disponívies para formar ligações cruzadas físicas), $\mathbf{K}$ é a constante de equilíbrio do processo de formação de ligações cruzadas, a é o fator de não-idealidade (reflete o quanto a rede formada difere de um elastômero ideal), e $\alpha, v$ e $\beta$ são variáveis dependentes, dadas respectivamente pelas equações 2 a 4 .

$\alpha=\mathbf{K} \mathrm{N} f(1-\alpha)^{2}$

onde $\mathrm{N}$ é o número de moles de polímero por unidade volumétrica e $\alpha$ representa a fração de grupos funcionais que reagiu.

$v=(1-\alpha+\alpha v)^{f-1}$
$\beta=(f-1) \alpha v /(1-\alpha+\alpha v)$

O significado físico de $v$ está ligado ao conceito de cadeia elasticamente ativa. Um grupo funcional de uma determinada macromolécula tem probabilidade, numericamente representada por $v$, de não contribuir para a elasticidade do gel. Um grupo funcional é considerado inativo quando não reage ou, quando reage mas não se liga a outros três grupos funcionais de ramos poliméricos infinitos 27,28 .

No presente trabalho, o modelo teórico de Clark e RossMurphy ${ }^{26}$ foi ajustado aos dados experimentais obtidos para géis físicos mistos de agarose e de goma guar, submetidos a testes de compressão uniaxial. Considerando-se a razão de Poisson de 0,5 e a relação $E=3 G$ entre o módulo de Young e o módulo de cisalhamento, os módulos de Young determinados experimentalmente para composições diversas de misturas de soluções de agarose e de goma guar foram utilizados nas estimativas. Os parâmetros $\boldsymbol{f}, \mathbf{K}$ e a foram analisados através de testes estatísticos.

\section{PARTE EXPERIMENTAL}

Agarose, doada pela CIALGAS - Companhia Industrial de Algas (Taboão da Serra, SP), foi usada como recebida. A viscosidade intrínseca, $[\eta]=3,24 \mathrm{dl} / \mathrm{g}$, foi determinada em viscosímetro do tipo Ostwald em $0,75 \mathrm{M}$ de $\mathrm{NaSCN}$, a $35^{\circ} \mathrm{C}$. O peso molecular viscosimétrico médio, $\overline{\mathrm{M}}_{v}=1,23 \times 10^{5}$, foi calculado através da relação de Mark-Houwink, tomando os valores de $\mathrm{K}=0,07$ e de $\mathrm{a}=0,72$ da literatura ${ }^{29}$.

A goma guar, fornecida pela Dowell Schumberger (Tulsa, USA) foi purificada conforme descrito previamente ${ }^{11}$. Sua viscosidade intrínseca, $[\eta]=13,6 \mathrm{dl} / \mathrm{g}$, foi determinada em água a $25^{\circ} \mathrm{C}$, de acordo com o procedimento acima. A relação de Mark-Houwink forneceu o valor de $\overline{\mathrm{M}}_{v}=19,9 \times 10^{5}$, a partir dos valores ${ }^{30}$ de $\mathrm{K}=3,8 \times 10^{-4}$ e de $\mathrm{a}=0,723$.

Géis mistos de agarose e de goma guar, de composição 1:1 e de concentrações totais em polímero variadas, foram obtidos pela mistura a $85^{\circ} \mathrm{C}$, de volumes apropriados de soluções dos polissacarídeos puros a $1 \%(\mathrm{p} / \mathrm{p})$. As misturas eram transferidas para tubos de vidro de $17 \mathrm{~mm}$ de diâmetro e $150 \mathrm{~mm}$ de comprimento. Após gelificação a temperatura ambiente, 5 corpos de prova de $17 \mathrm{~mm}$ de altura foram cortados e imersos em água a $25^{\circ} \mathrm{C}$ durante uma noite. Os corpos de prova perfeitos foram submetidos a testes de compressão uniaxial em Máquina Instron, modelo TM-M, equipado com célula CTM, a $0,5 \mathrm{~cm} /$ $\min$ e a $25^{\circ} \mathrm{C}$.

Para o processo de estimação dos parâmetros característicos dos géis de mistura, um algorítimo foi elaborado ${ }^{31}$ em linguagem FORTRAN IV.

\section{RESULTADOS E DISCUSSÃO}

As redes tridimensionais de géis de biopolímeros diferem daquelas formadas por ligações cruzadas químicas, em sistemas de polímeros sintéticos. Enquanto que as redes tridimensionais nos géis químicos são originadas por reações que se passam em certos pontos das cadeias, a formação de um gel físico envolve a associação não mais de pontos, mas de segmentos ou regiões ordenadas das macromoléculas, através de interações bem mais fracas e reversíveis. Além do componente macromolecular, responsável por sua elasticidade, os géis são constituídos de solvente aquoso, sempre em excesso.

A descrição quantitativa da relação módulo-concentração para géis de biopolímeros proposta por Clark e Ross-Murphy ${ }^{26}$ foi baseada em modelos teóricos sugeridos anteriormente para géis químicos ideais, como o de Flory ${ }^{16}$ e Stockmayer ${ }^{17}$, e para géis de biopolímeros, como o de Hermans ${ }^{25}$.

$\mathrm{O}$ parâmetro $f$ da equação 1 , a funcionalidade, é definido pela ciência de polímeros. Moléculas bifuncionais (monômeros), de $f=2$, dão origem a polímeros lineares. A presença de monômeros polifuncionais, $\boldsymbol{f}>2$, quase sempre leva à formação de estruturas químicas de dimensões macros-cópicas, às quais a denominação de rede tridimensional química é aplicada. Em todos os modelos teóricos citados, $f$ representa a funcionalidade de uma macromolécula linear, ou o número de grupos funcionais que possui e que estão disponíveis para a formação de ligações covalentes ou de interações físicas adicionais, que levarão à formação de uma rede tridimensional química ou física, respectivamente.

A constante $\mathrm{K}$ foi utilizada inicialmente na dedução do modelo de Hermans ${ }^{25}$, que considerou como um equilíbrio (interação $\rightleftharpoons$ dois sítios livres) o processo de formação de ligações cruzadas físicas.

Clark e Ross-Murphy ${ }^{26}$ utilizaram os métodos da teoria das ramificações para quantificar o número de cadeias elasticamente ativas e formular a relação módulo-concentração. Enquanto que a elasticidade de borrachas ideais é essencialmente entrópica (o módulo é proporcional à temperatura absoluta), na 
maioria dos géis físicos as contribuições entálpicas são muito importantes. Assim, a suposição da contribuição de RT por mol de cadeias elasticamente ativas foi abandonada e o parâmetro de não-idealidade a foi introduzido, considerando-se aRT como a contribuição real. O produto aRT representa energia livre, e a, portanto, envolve contribuições entálpicas e entrópicas.

A estimação dos parâmetros associados ao modelo de Clark e Ross-Murphy foi realizada empregando-se uma rotina computacional baseada no princípio da máxima verossimilhança $^{32}$, generalizada em trabalho mais recente ${ }^{33}$. Pelo princípio da máxima verossimilhança, os valores estimados para os parâmetros devem ser aqueles que levem aos valores teóricos mais próximos possíveis dos valores observados experimentalmente.

A função objetivo $\mathrm{S}$ pode ser expressa em termos do módulo de Young, E, e da variança $\delta^{2}$.

$$
\mathrm{S}=\frac{\sum_{\mathrm{i}=1}^{\mathrm{N}}\left(\overline{\mathrm{E}}_{\mathrm{i}}^{\text {exp }}-\mathrm{E}_{\mathrm{i}}^{\mathrm{calc}}\right)^{2}}{\delta \frac{2}{\mathrm{E} i}}
$$

onde $\overline{\mathrm{E}}_{\mathrm{i}}$ exp é a média dos valores experimentais obtidos para o módulo de Young da iésima experiência ou concentração total, de uma série de $\mathrm{N}=6$ experiências ou concentrações totais da mistura, e $\mathrm{E}_{\mathrm{i}}^{\mathrm{cal}}$ é o valor correspondente ao módulo de Young calculado pelo modelo.

A rotina computacional leva aos parâmetros desejados e aos seus desvios-padrão, pela minimização da função $S$.

A tabela 1 mostra os valores médios do módulo de Young, $\overline{\mathrm{E}}$, e da variança, $\delta_{\overline{\mathrm{E}}}^{2}$, para os géis mistos de agarose e goma guar.

Tabela 1. Módulos de Young e varianças correspondentes para géis mistos de agarose e goma guar.

\begin{tabular}{ccc}
\hline $\mathrm{C}(\% \mathrm{p} / \mathrm{p})$ & $\overline{\mathrm{E}} \times 10^{-4}(\mathrm{~Pa})$ & $\delta_{\overline{\mathrm{E}}}^{2}\left(\times 10^{-6}\right)$ \\
\hline 0,5 & 0,37 & 0,018 \\
0,6 & 0,43 & 0,011 \\
0,7 & 0,59 & 0,111 \\
0,8 & 0,79 & 0,781 \\
1,0 & 1,53 & 0,154 \\
1,4 & 2,33 & 1,360 \\
\hline
\end{tabular}

Conforme sugerido na literatura ${ }^{34,35}$, os parâmetros $\mathbf{K}$ e a foram otimizados para vários valores de $f$. O coeficiente de correlação entre $\mathbf{K}$ e a aumenta em função do valor de $f$, como mostrado na tabela 2 .

A ordem de grandeza alcançada pelos coeficientes de correlação indica que os parâmetros $\mathbf{K}$ e a são altamente correlacionados, o que não propicia uma boa estimação. Este resultado mostra que a estratégia de fixar-se $f$ não é suficiente para reduzir a correlação entre $\mathbf{K}$ e $\mathbf{a}$.

A tabela 3 apresenta os resultados de $\mathbf{K}$, a e $\mathrm{C}_{\mathrm{o}}$, assim como os valores da função objetivo, $\mathrm{S}$, para os géis mistos de agarose-goma guar, para cada uma das funcionalidades consideradas. $\mathrm{O}$ peso molecular viscosimétrico médio da agarose, $\mathrm{M}_{\mathrm{v}}=1,2 \times 10^{5}$, foi considerado nos cálculos. Pode-se verificar que a função objetivo apresentou valores na faixa 64,39 a 72,51 , valores esses bem mais altos do que aqueles determinados para géis puros de agarose ${ }^{31}$, para os quais a função objetivo variou de 5,03 a 11,55 , no intervalo $3 \leq f \leq 100$. É possível que essa diferença na qualidade do ajuste esteja relacionada a alterações no mecanismo de gelificação da agarose na presença da goma guar.
Tabela 2. Coeficientes de correlação entre $\mathbf{K}$ e $\mathbf{a}$.

\begin{tabular}{rr}
\hline$f$ & coeficientes de correlação \\
\hline 3 & 0,9609 \\
10 & 0,9546 \\
25 & 0,9549 \\
50 & 0,9649 \\
75 & 0,9750 \\
100 & 0,9804 \\
200 & 0,9836 \\
500 & 0,9898 \\
1000 & 0,9946 \\
1500 & 0,9968 \\
2000 & 0,9977 \\
\hline
\end{tabular}

A qualidade do ajuste pode ser melhor avaliada através da representação gráfica dos resultados de módulo de Young obtidos experimentalmente, e os que foram obtidos através do modelo teórico. A figura 1 permite observar-se que o modelo foi capaz de representar bem os resultados experimentais, apesar dos valores da função objetivo.

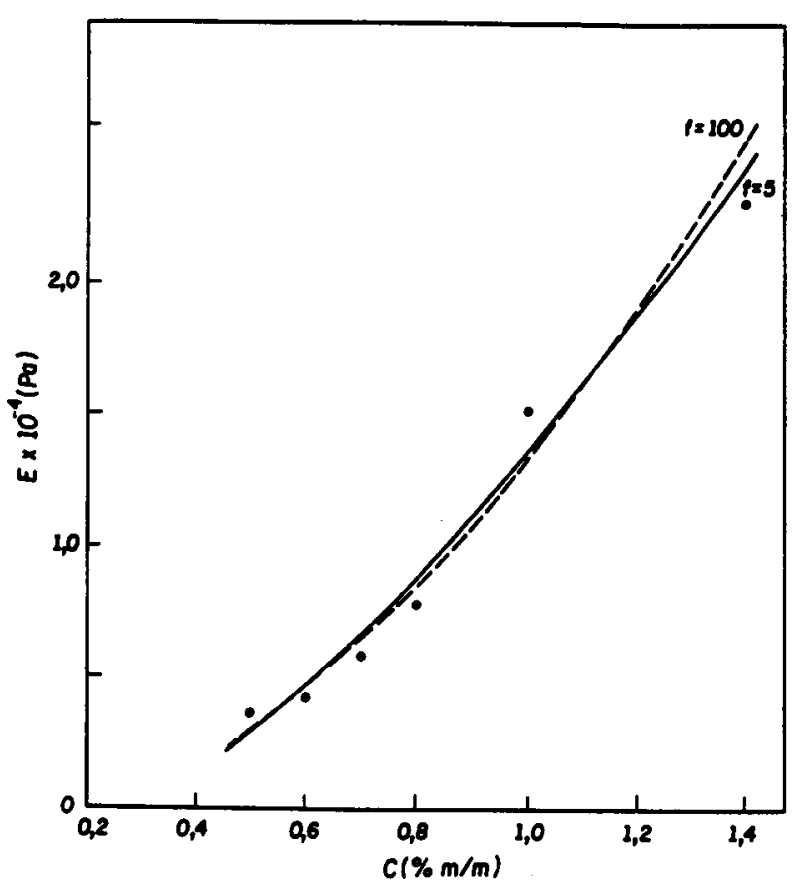

Figura 1. Variação do módulo de Young com a concentração total de polímero (†) e as curvas estimadas pelo modelo de Clark e Ross-Murphy, para géis mistos de agarose e de goma guar, de composição 1:1.

De acordo com a tabela 3, pode-se verificar que a função objetivo diminuiu com o aumento de $f$, o que a grosso modo representaria uma melhoria no ajuste. Assim, um ajuste "melhor" seria o obtido para $f=2000$. Contudo, nessa funcionalidade, os desvios-padrão observados para os parâmetros $\mathbf{K}$ e a foram altíssimos, maiores até do que os próprios valores dos parâmetros. Obviamente, isto torna inconsistentes as estimativas para $f=2000$. 
Tabela 3 - Estimativa dos parâmetros $\mathbf{K}, \mathbf{a}, \mathrm{C}_{\mathrm{o}}$ e da função objetivo $\mathrm{S}$, para os géis mistos de agarose e goma guar.

\begin{tabular}{|c|c|c|c|c|}
\hline$f$ & $\begin{array}{c}\mathbf{K} \\
\left(\mathrm{m}^{3} / \mathrm{mol}\right)\end{array}$ & $\mathbf{a}$ & $\begin{array}{c}\mathrm{C}_{\mathrm{o}} \\
(\% \mathrm{p} / \mathrm{p})\end{array}$ & $S$ \\
\hline 3 & $(4,85 \pm 0,86) \times 10$ & $49,60 \pm 11,06$ & 0,165 & 70,31 \\
\hline 5 & $(6,17 \pm 1,02)$ & $24,90 \pm 5,24$ & 0,173 & 72,48 \\
\hline 10 & $(9,89 \pm 1,64) \times 10^{-1}$ & $15,08 \pm 3,19$ & 0,171 & 72,51 \\
\hline 25 & $(1,37 \pm 0,26) \times 10^{-1}$ & $9,92 \pm 2,33$ & 0,158 & 70,10 \\
\hline 50 & $(3,48 \pm 0,70) \times 10^{-2}$ & $7,91 \pm 2,16$ & 0,146 & 68,11 \\
\hline 75 & $(1,59 \pm 0,41) \times 10^{-2}$ & $7,11 \pm 2,17$ & 0,140 & 67,19 \\
\hline 100 & $(9,17 \pm 2,60) \times 10^{-3}$ & $6,64 \pm 2,22$ & 0,135 & 66,65 \\
\hline 200 & $(2,45 \pm 0,90) \times 10^{-3}$ & $5,79 \pm 2,42$ & 0,124 & 65,71 \\
\hline 500 & $(4,28 \pm 2,22) \times 10^{-4}$ & $5,03 \pm 2,87$ & 0,113 & 64,95 \\
\hline 1000 & $(1,15 \pm 0,80) \times 10^{-4}$ & $4,58 \pm 3,39$ & 0,105 & 64,60 \\
\hline 1500 & $(5,34 \pm 4,43) \times 10^{-5}$ & $4,34 \pm 3,78$ & 0,100 & 64,45 \\
\hline 2000 & $(4,55 \pm 17,00) \times 10^{-5}$ & $2,82 \pm 11,10$ & 0,066 & 64,39 \\
\hline
\end{tabular}

Uma outra fonte de informação sobre os parâmetros estimados pode ser obtida através da utilização do teste-t. Este teste avalia a significância de um determinado valor estimado, dado o seu desvio-padrão ${ }^{36}$. Para os géis mistos de agarose e goma guar, onde o grau de liberdade (número de experimentos número de parâmetros) foi determinado em 4, considerando-se um grau de confiança de $95 \%$, o índice-t foi determinado como igual a 2,78. A análise comparativa do índice-t e das razões entre os valores estimados de $\mathbf{K}$ e $\mathbf{a}$ e os seus desvios-padrão correspondentes, $\delta \mathbf{K}$ e $\delta \mathbf{a}$, mostra que para $\boldsymbol{f} \cdot 200$, as razões $\mathbf{K} / \delta \mathbf{K}$ e $\mathbf{a} / \delta \mathbf{a}$ apresentam-se menores do que o índice-t. De acordo com a interpretação estatística, este resultado indica que para $\boldsymbol{f} \cdot 200$, os parâmetros $\mathbf{K}$ e $\mathbf{a}$ apresentam valores matematicamente inconsistentes.

O resultado da estimativa dos parâmetros dos géis mistos de agarose e goma guar foram analisados também pelo teste F. Este teste, através do número total de experimentos realizados e do grau de liberdade do sistema, permite que se determine se, estatisticamente, as estimativas podem ou não ser diferenciadas $^{36}$. Para os géis considerados neste trabalho, o número total de experimentos realizados foi de 21 e o grau de liberdade foi igual a 4; o valor encontrado para o índice $\mathrm{F}$ foi de 3,47 . A razão entre a maior função objetivo $(f=10)$ e a menor $(f=$ $100)$ é de 1,09 , um valor menor do que o encontrado para o índice F. Este resultado mostra que os valores estimados de $\mathbf{K}$ e a nos dois casos representam igualmente bem os dados experimentais.

Embora o modelo teórico utilizado não seja capaz de definir matematicamente o melhor conjunto de parâmetros, os valores calculados para $C_{o}$ apresentaram consistência física. Apesar de não ter sido possível determinar-se experimentalmente a concentração mínima de gelificação, pôde-se observar visualmente a formação de gel macroscópio para misturas 1:1 de agarose e goma guar de concentração total $0,2 \%$.

Os valores estimados para o fator de não-idealidade, a, apresentaram-se na faixa 49,60 a 6,64 , para funcionalidades entre 3 e 100. Sabe-se que o valor de a aproxima-se de 1 quando as cadeias entre as zonas de junção, isto é, cadeias em conformação desordenada, apresentam comportamento semelhante ao das cadeias entre as ligações cruzadas na borracha ideal. Conforme as cadeias entre as zonas de junção adquirem restrições conformacionais, o valor de a cresce ${ }^{37}$. Dessa forma, os resultados obtidos para a podem ser considerados coerentes, já que a elasticidade de uma cadeia de polissacarídeo entre as zonas de junção está longe de ser considerada semelhante àquela de uma borracha ideal.

Embora a constante de equilíbrio $\mathrm{K}$ tenha sido fundamental na obtenção do modelo proposto por Clark e Ross-Murphy, ora empregado neste trabalho, a análise de seus valores deve ser feita com certa precaução. Na verdade, $\mathbf{K}$ foge à definição de uma constante de equilíbrio ${ }^{38}$, já que em géis físicos de polissacarídeos o número de ligações intermoleculares aumenta continuamente com o tempo. Por esse motivo, os valores de $\mathbf{K}$ obtidos pelo processo de estimação não serão discutidos.

Uma outra maneira de estimar-se os parâmetros dos géis de agarose-goma guar foi realizada a partir da utilização do peso molecular da agarose, $1,2 \times 10^{5}$, e considerando-se apenas a concentração de agarose nos géis. A tabela 4 apresenta os resultados obtidos para as funcionalidades 3 e 100. Pode-se observar que a função objetivo nesses dois casos apresentou os mesmos valores que os observados na tabela 3 , o que significa que o ajuste foi de qualidade igual.

É possível que a dificuldade encontrada para a definição do melhor conjunto de parâmetros $\boldsymbol{f}, \mathbf{K}$ e a esteja relacionada ao fato de o modelo de Clark e Ross-Murphy ${ }^{26}$ não levar em consideração o comportamento de fases das soluções gelificantes de biopolímeros. Sabe-se que alguns géis de biopolímeros mesmo sendo constituídos por um componente macromolecular apenas (como é o caso da agarose ${ }^{9-11}$ ), apresentam separação de fases durante a sua formação. Outro fator que dificulta a aplicação do modelo é a polidispersão da amostra polimérica ${ }^{34}$, casualidade usualmente encontrada em polímeros naturais, e só superada após fracionamento.

Tabela 4 - Estimativa de $\mathbf{K}$, a e $\mathrm{C}_{\mathrm{o}}$ para géis de agarose-goma guar.

\begin{tabular}{rcccc}
\hline $\boldsymbol{f}$ & $\begin{array}{c}\mathbf{K} \\
\left(\mathrm{m}^{3} / \mathrm{mol}\right)\end{array}$ & $\mathbf{a}$ & $\begin{array}{c}\mathrm{C}_{\mathrm{o}} \\
(\% \mathrm{p} / \mathrm{p})\end{array}$ & $\mathrm{S}$ \\
\hline 3 & $(9,71 \pm 1,73) \times 10$ & $99,21 \pm 22,12$ & 0,082 & 70,31 \\
100 & $(1,83 \pm 0,52) \times 10^{-2}$ & $13,29 \pm 4,44$ & 0,067 & 66,65 \\
\hline
\end{tabular}


Com relação à concentração mínima de gelificação, $\mathrm{C}_{0}$, pode-se notar que os valores obtidos correspondem à metade daqueles observados na tabela 3 , onde a concentração de polímero utilizada foi a concentração total de agarose e de goma guar presentes na mistura. Uma observação interessante, que merece ser destacada, diz respeito aos resultados da tabela 3. Os valores calculados para $C_{0}$, no intervalo $f=3-100$, mantiveram-se entre 0,165 e $0,135 \%$, o que demonstra sua consistência física, levando-se em conta que a formação de gel macroscópico para misturas 1:1 de agarose e de goma guar foi observada visualmente para concentrações totais tão baixas quanto $0,2 \%$. Os valores menores, calculados para $\mathrm{C}_{\mathrm{o}}$, corroboram a proposição de que a goma guar de fato participa do mecanismo de gelificação da mistura agarose e goma guar ${ }^{39}$, e não exerce apenas o papel de "carga" inerte nos interstícios da rede do polissacarídeo gelificante agarose.

\section{CONCLUSÃO}

Os resultados discutidos neste trabalho mostram que o modelo matemático utilizado é capaz de representar bem os dados experimentais obtidos para géis binários submetidos a testes de compressão uniaxial. Entretanto, como já havia sido verificado anteriormente para géis puros de agarose ${ }^{31}$, o modelo de Clark e Ross-Murphy não é capaz de definir o melhor conjunto de parâmetros para o sistema, mas pode vir a constituir-se numa base para a elaboração de modelos mais precisos, dada à coerência dos resultados que apresenta.

\section{REFERÊNCIAS BIBLIOGRÁFICAS}

1. Flory, P. J.; Faraday Discuss. Chem. Soc. 1974, 57, 7.

2. Callister, S.; Keller, A.; Hikmet, R. M.; Makromol. Chem., Macromol. Symp. 1990, 39, 19.

3. Ferry, J. D.; Viscoelastic Properties of Polymers, John Wiley \& Sons, Inc., New York, 1970, p 365.

4. Graessley, W. W.; Adv. Polym. Sci. 1974, 16, 1.

5. Tanaka, T.; In Structure and Dynamics of Biopolymers; Nicolini, C., Ed.; Martinus Nijhoff Publishers: Dordrecht, 1987, p 237.

6. Rees, D. A.; Pure Appl. Chem. 1981, 53, 1.

7. Arnott, A.; Fulmer, A.; Scott, W. E.; Dea, I. C. M.; Moorhouse, R; Rees, D. A. J.; Mol. Biol. 1974, 90, 269.

8. Pines, E.; Prins, W.; Macromolecules 1973, 6, 888.

9. San Baggio, P. L.; Bulone, D.; Emanuele, A.; Madonia, F.; Di Stefano, L.; Giacomazza, D.; Trapanese, M; Palma-Vitorelli, M. B.; Palma, M. U.; Makromol. Chem., Macromol. Symp. 1990, 40, 33.

10. Emanuele, A.; Di Stefano, L.; Giacomazza, D.; Trapanese, M.; Palma-Vitorelli, M. B.; Palma, M. U.; Biopolymers 1991, 31, 859 .

11. Andrade, C. T.; Garcia, R. B.; Abritta, T.; Polym. Bull. 1991, 27, 297.

12. Morris, E. R.; In Gums and Stabilisers for the Food
Industry 2; Phillips, G. O.; Wedlock, D. J.; Wedlock, D. J.; Williams, P. A., Ed.; Pergamon Press: Oxford, 1983; p 57.

13. Ross-Murphy, S. B.; Rheological Methods; King's College: London, 1992; p 5.

14. Clark, A. H.; In Polymers, Gels and Colloids; Dickinson, E., Ed.; RSC: London, 1991; p 322.

15. Clark, A. H.; In Food Structure and Behaviour; Blanshard, J. M. V.; Lillford, P. J., Ed.; Academic Press: London, 1987; p 13.

16. Flory, P. J.; Faraday Discuss. Chem. Soc. 1941, 63,123.

17. Stockmayer, W. H. J. Chem. Phys. 1943, 11, 45.

18. Allen, G.; Holmes, P. A.; Walsh, D. J.; Faraday Discuss. Chem. Soc. 1974, 57, 19.

19. Peniche-Covas, C. A. L.; Dev, S. B.; Gordon, M.; Judd, M.; Kajiwara, K.; Faraday Discuss.Chem. Soc. 1974, 57, 165.

20. Queslel, J. P.; Mark, J. E.; In Encyclopedia of Polymer Science and Engineering; Mark, H. F.; Bikales, N. M.; Overberger, C. G.; Menges, G.; Kroschwitz, Ed., John Wiley \& Sons: New York, 1986; vol. 5, p 365.

21. Price, G. J.; Siow, K. S.; Guillet, J. E.; Macromolecules 1989, 22, 3116 .

22. Nishinari, K.; Koide, S.; Ogino, K. J.; Physique 1985, 46, 793.

23. Ross-Murphy, S. B.; F. Hydrocol. 1987, 1, 485.

24. Clark, A. H.; Ross-Murphy, S. B.; Adv. Polym. Sci. 1987, $83,56$.

25. Hermans, J. J.; Polym. Sci. A 1965, 3, 1859.

26. Clark, A. H.; Ross-Murphy, S. B.; Brit. Polym. J. 1985, $17,164$.

27. Dobson, G. R.; Gordon, M.; J.; Chem. Phys. 1965, 43, 705.

28. Gordon, M.; Ross-Murphy, S.B.; Pure Appl. Chem. 1975, $43,1$.

29. Rochas, C.; Lahaye, M.; Carbohydr. Polym. 1989, 10, 289.

30. Baines, Z. V.; Morris, E. R.; F. Hydrocoll. 1987, 1, 197.

31. Garcia, R. B.; Lobão, M. W. N.; Andrade, C. T.; Polym. Reaction Eng. 1994, 2, 1.

32. Anderson, T. F.; Abrams, D. S.; GrensII, E. A.; AIChE J. 1978, 24, 20.

33. Noronha, F. B.; Pinto, J. C.; Monteiro, J. L.; Lobão, M. W. N.; Manual Estima - Um Pacote Computacional para Estimação de Parâmetros e Projetos de Experimentos; COPPE-UFRJ: Rio de Janeiro, 1991; p 1.

34. Clark, A. H.; Gidley, M. J.; Richardson, R. K.; RossMurphy, S. B.; Macromolecules 1989, 22, 346.

35. Watase, M.; Nishinari, K.; Clark, A. H.; Ross-Murphy, S. B.; Macromolecules 1989, 22, 1196.

36. Himmelblau, D. M.; Process Analysis by Statistical Methods; John Wiley \& Sons: New York, 1970; p 436.

37. Ross-Murphy, S. B.; McEvoy, H.; Brit. Polym. J. 1986, 18, 2.

38. Maron, S. H.; Prutton, C. F.; Principles of Physical Chemistry, The Macmillan Company: New York, 1965; p 232.

39. Garcia, R. B.; Lopes, L.; Andrade, C. T.; Fresenius' J. Anal. Chem. 1992, 344, 510. 